\title{
Improvement of road traffic management system on the basis of mathematical and simulation modeling
}

\author{
Denis Lomakin ${ }^{1, *}$, Andrei Bodrov ${ }^{1}$, Maksim Kulev${ }^{1}$, and Andrei Kulev ${ }^{1}$ \\ ${ }^{1}$ Orel State University, 302026 Orel, Komsomolskaya st. 95, Russian Federation
}

\begin{abstract}
A mathematical model of the "Driver-Vehicle-RoadEnvironment" system is substantiated in the form of a multidimensional random process with a large number of components based on a structural approach which is a basic algorithm for subsequent modeling of traffic flows on a road network.
\end{abstract}

\section{Introduction}

Modern complex systems, in this case, "Driver- Vehicle-Road-Environment” system (DVRE), are characterized by the presence of a large number of functionally different elements acting according to different physical principles [1-3]. Setting the task of analyzing rare events such as failures (road congestions, discomfort traffic), one should not limit oneself to a deterministic approach, which in many situations is insensitive to these events, when constructing a mathematical model of a system [4-7]. It is necessary to identify all conceivable accidents affecting the action of the system, that is, to describe each element of the system at the accepted level of detail by some random process. The mathematical model of the entire system is obtained in the form of a multidimensional random process with a large number of components [6, 8-10]. The most rational way of constructing a mathematical model of the DVRE system is the aggregate (structural) approach [11].

\section{Theory}

The behavior of the element (driver, vehicle, road and environment) is described by a random process $Z(t)$. The value of $Z(t)$ at point $t$ is called the (internal) state of the element of the DVRE system at point $t$. It is assumed that the elements can serve as a mathematical model of the system behavior as a whole and also its subsystems. Certain restrictions of laws according to which some elements affect others are needed for the possibility of effective interaction of the elements or subsystems that describe the elements. A circuit with discrete signal transmission is a promising option for such restrictions: an element

\footnotetext{
*Corresponding author: forstudentwork@mail.ru
} 
operates independently of other elements until a signal from any other element arrives at its input. This circuit allows building a model of the DVRE system [3, 12-16].

The possibility of real application of the circuit with discrete signal transmission is based on the fact that the DVRE system is designed to perform operations consisting of elementary operations chains assigned to an element or groups of elements ("channels") of the system. When creating a model of the DVRE system, it is necessary to try to protect the channel from the effect of outside influence when performing the function assigned to it. In the ideal case, the connection of this channel with other parts of the system occurs only at the moments of receiving the task, which corresponds to the input signal, and the issuance of the finished result (output signal). Signals are also transmitted if it is necessary to correct a task, to load additional information, to redirect the channel to perform another task, but, in general, also at discrete time instants [17-19].

Let us now imagine the effect of the DVRE system in the conditions of component failures (violation of traffic rules by the driver, vehicle failure, traffic light failure, road surface condition, adverse weather conditions, vehicle crash, etc.). Conditions under which the given operation cannot be performed by this channel may be provided. If the cause is a failure of an element that is part of the channel itself, this can be taken into account by a corresponding change in the internal state of the element describing the channel; if the reason is the failure of other elements of the system, then in the mathematical model, transmission should be provided from the corresponding elements of the signal, by which the data execution by the operation channel stops(or, say, the speed of the operation is changed, in particular, a change in the direction of movement or a decrease in the speed of the car) [19].

To build a random-process realization means to set up an algorithm for a given source of randomness (for example, a random number generator) that assigned any output of the source with the function $\xi(t), t \in\{t\}$. Then, by $\omega$, we should understand everything that we took from the input of the source of randomness, say, a fixed number of random numbers or random numbers $\omega_{1} \ldots \ldots, \omega_{v}$, where $\mathrm{v}$ is the first moment of some event determined by the first $v$ random numbers ( for example, the first moment when $\omega^{2}{ }_{1}+\ldots+\omega^{2}{ }_{v} \geq N$ ). Thus, the general definition of a random process as a function of two variables $t$ and $\omega$, upon deeper consideration, is fully consistent with the goals of constructing models of real phenomena.

The concept of a terminating random process reflects the finiteness in time of real operations in systems $[6,20]$. An example of a terminating random process is the Poisson process with $\lambda$ parameter with a gap at the moment of reaching the level $n \geq 1$. As the point $\omega$, we can take the set of $\mathrm{n}$ random variables $\omega_{1} \ldots, \omega_{n}$ exponentially distributed with $\lambda$ parameter, where $\omega_{1}$ is the first jump time; $\omega_{i}, t \geq 2$ is the time between the $(i-1)$ - th and $i$ - th jump of the trajectory of the Poisson process. Thus, in this case, we can explicitly express $\xi(t, \omega)$ and $\tau(x \leq 0 \omega)$ in terms of $\omega$ : if $E(x)=1$ for $x>0, E(x)=0$ for $x \leq 0$, then in the notation $s_{i}=\omega_{1}+\ldots+\omega_{i}$.

$$
\tau(\omega)=\omega_{1}+\ldots+\omega_{n} ;-\xi(t-\omega)=\sum_{i=1}^{n} E\left(t-s_{1}\right), 0 \leq t<\tau(\omega)
$$

The concept of the terminating process naturally extends to the case of determining it in the half-interval $\left[t_{0}, t_{0+\tau}\right]$. The terminating random process could be taken as a fairly general model of the action of an element of the complex DVRE system provided that it is autonomous (independent of other elements). To take into account the possibility of mutual influences, two additional elements should be introduced: output signals sent by a system element, and a change in its state under the influence of input signals. Entering the output signals is the simplest. Suppose that the trajectory of the terminating random process $\xi(t)$ with probability 1 is indiscrete in the half-interval $[0, \tau]$ in some topology of the space $\mathrm{Z}$. 
We define the set $Y$ of possible output signals y. Put into correspondence a closed set $Z_{y} \subset Z$ to each $y \in Y$, and suppose that $Z_{y}$ disjoint under a variety of $y \in Y$. Signals are sent as follows: if $t=\min \left\{s: \xi(s) \in Z_{y}\right\}$, then at time t the output signal y is sent. Otherwise, $y$ signal is sent at the moment of the first arrival of $\xi(t)$ in the set of states $Z_{y}$.

The reaction of the element to the input signals was set as follows: by arranging in time $t_{i}<t_{2}<\ldots$ moments of the input of the signal element and, for definiteness, setting $t_{0}=0<t_{1}$, for any $n \geq 0$ at the moment $t_{n}$, the conditional trajectory of the random process $z(t)$, which describes the behavior of this element in the half-interval $\left[t_{n}, t_{n}+\tau_{n}\right]$, is determined. This trajectory is realized until the next input signal arrives, more precisely, until the min $\left\{t_{n}+1\right.$, $\left.t_{n}+\tau_{n}\right\}$ moment. If $t_{n}+1<t_{n}+\tau_{n}$, then at the moment $t_{n}+1$ the conditional trajectory is canceled and a new conditional trajectory is constructed, depending on the incoming signal and on the previous behavior of the element of the DVRE system.

Let $X=\{x\}$ be the set of possible values of the input signal supplied to the input of a given element of the system. If for some $n \geq 1 t_{n-1}+\tau_{n}$, then this state is considered the moment of termination of the functioning of an element of the DVRE system. If $t_{n}<t_{n-1}+\tau_{n-1}$, then from the th moment the conditional trajectory of the $z(t)$ process is determined. Namely, let $(\xi(t) \tau)=(\xi(t, \omega, X, z(s)), \tau(\omega, x, z,(s))$ be a family of terminating random processes depending on $x, z(s)$ as a function of parameters, and $\left(\xi_{0}(t), \tau_{0}\right)$ is another terminating random process; $\omega_{0}, \omega_{1} \omega_{2}, \ldots$ is a sequence of independent elements of the space $\Omega$. First, the conditional trajectory of the state of the DVRE system $z(t)$ in the halfinterval $\left[0, \tau_{0}\right]$ is determined as follows:

$$
z(t)=\xi_{o}\left(t, \omega_{0}\right), 0 \leq t<\tau_{0}=\tau_{0}\left(\omega_{0}\right) .
$$

If $t_{1} \geq \tau_{0}$, then the trajectory is confirmed by formula (2) and at the moment $\tau_{0}$ the functioning of the element ceases. Otherwise, when the $x_{1}$ signal arrives at $t_{1}$ time, we assume:

$$
Z(t)=\xi_{0}(t), 0 \leq t<t_{1} ; Z(t)=\xi_{0}\left(t-t_{1}, \omega_{1}, x_{1}, z\left(t_{1}\right)\right), t_{1} \leq t<t_{1}+\tau\left(\omega_{1}, x_{1}, z\left(t_{1}\right)\right) .
$$

The $\mathrm{z}(\mathrm{t})$ trajectory segment given by formula (3) is confirmed for $0 \leq t<t_{1}$, conditional for $t_{1} \leq t<t_{2}$, i.e., (3) are actually fulfilled for $0 \leq t<\min \left\{t_{2}, t,+\tau\left(\omega_{1}, X_{1}, z\left(t_{1}\right)\right)\right\}$.

Let the trajectory of the internal state of an element be generally defined as a $z(t)$ function in the half-interval $\left[0, t_{n}+\tau_{n}\right]$. Then in the half-interval $\left[0, t_{n}\right]$ this trajectory is confirmed. If $t_{n}+1 \geq t_{n}+\tau_{n}$, it is also confirmed in the entire half-interval [0, $\left.t_{n}+\tau_{n}\right]$, and at the $t_{n}+\tau_{n}$ moment, the functioning of the unit ceases. If $t_{n}+1<t_{n}+\tau_{n}$, then the previously constructed trajectory is confirmed in the half-interval $\left[0, t_{n}+1\right]$ and the conditional (that is, subsequently confirmed until the next signal arrives) $z(t)$ trajectory is determined in the half-interval $\left[t_{n}+1, t_{n}+\tau_{n}+1\right]$, where:

$$
\begin{aligned}
& \tau_{n+1}=\tau\left(\omega_{n+1}, z\left(t_{n+1}\right)\right), \\
& z(t)=\xi\left(t-t_{n+1}, \omega_{n+1}, x_{n+1}, z\left(t_{n+1}\right)\right), \\
& t_{n+1} \leq t<t_{n+1}+\tau_{n+1} .
\end{aligned}
$$

where $x_{n}+1$ is the signal arriving at the input of the DVRE system at the moment $t_{n}+1$

Suppose that the integrals in probability spaces used to find the distributions of these functional exist. Also, the finiteness condition with probability 1 of the number of signals (both input and output) in any interval $\left(0, t^{*}-e\right)$, where $e>0, t^{*}$ is the moment when the unit stops functioning [at $t^{*}=\infty$ in the interval $(0, T)$ ] will not be specifically stipulated.

We give an example of using the proposed mathematical model in the framework of Intelligent Transport Systems. As the object of study, we take traffic management at the 
road intersections of the city of Orel. Traffic flows, when approaching the crossing, can be divided in different directions, depending on the permitted driving directions, i.e. tasks of the 1st, 2nd, ..., mth type arise for the DVRE system. The relative importance of various tasks varies depending on the situation and is indicated by a control signal, i.e. fixed route of traffic flow. The process of traffic management at the crossing is described by a random process:

$$
z(t)=\left(v_{o}(t), v_{1}(t) \ldots, v_{m}(t), v_{m+l}(t), \xi(t)\right)
$$

where $v_{o}(t)$ is a parameter characterizing the system of priorities in force at the current time; $v_{i}(t), \quad 1 \leq i \leq m$, is the number of tasks of the $i$-th type that are in a waiting state at time; $v_{m}+1(t)$ is the type of work carried out at time $t$; $\xi(t)$ is the time remaining until the completion of this work.

The completion of work is indicated by the output $y=i$ signal, where $i$ is the type of work being performed. The y signal is sent at the moment when $Z(t)$ gets into the set of states $Z y=\left\{v_{m}+1(t)=y, \xi,(t)=0\right\}$. Input signals are of two types: non-controlling and controlling. Non-controlling $x$ signal with values $1, \ldots, m$ means the arrival of new work of the $x$-th type (discomfort traffic, traffic jam, etc.). The controlling signal changes the order of priority execution of the type of work, i.e. ITS, analyzing the difficulty encountered on the roadway, determines the ways of the priority driving direction.

An example of the application of the proposed model is the development of organizational and technical measures in a number of sections of the road network (RN) of the city of Orel. Verification of the effectiveness of the proposed organizational and technical measures was carried out through simulation.

Recently, simulation in the theory of traffic flows has become widespread, as it allows solving problems taking into account many parameters. Now there are many software products that can create analogues of urban transport networks. Among the many simulation software products, we can distinguish the following: such simulation environments as Aimsun (Spain), True (France), AutoMod (USA), AweSim (USA), eMPlant (Germany), as well as Arena (USA), PTVVisionVissim and PTVVisionVisum (Germany) systems. Similar modeling tools and software products are also present at domestic developers: ActorPilgrim, AGNES, GPSSWorld, RandModelDesigner and BMSTU.

The authors adopted PTVVisionVissim as the most common system of simulation modeling. The object of study is the intersection of Generala Rodina Street and Polesskaya Street.

The roadway on Generala Rodina Street has one lane in forward and backward direction. Oncoming traffic flows on the approach are separated by a marking line 1.1 (GOST P 51256-2011). The following traffic signs are installed (GOST P 52290-2004): 4.8.2 - Driving direction of vehicles with dangerous goods - straight ahead, 6.15.1 - Driving direction for trucks - straight ahead, 2.1 - Priority road, 5.11 .1 (5.19.2) - Pedestrian crossing sign.

Polesskaya Street has two lanes for direct and oncoming traffic. Turning traffic flows from it are separated by a marking line 1.3 (GOST P 51256-2011). In front of the crossing there is 2.4 "Give way" sign.

In accordance with the mathematical model presented earlier, as functions of the input $\mathrm{X}(\mathrm{t})$ parameter, we take the traffic intensity, the composition of the traffic flow, the geometric parameters of the intersection of the carriageways, and the presence of transport infrastructure at the crossing in question. 
The traffic intensity and composition of the traffic flow on the site was determined according to the methodology developed at the Service and Repair of Cars Department of our University.

The record of traffic intensity and composition of the traffic flow were kept according to the special registration form for each approach, where the number of vehicles of the corresponding types in each direction was recorded. Three measurements were made in the period from 7.00 a.m until 9.00 a.m, from 12.00 until 2.00 p.m and from 5.00 p.m until 7.00 p.m. Hourly traffic volume is defined as the average of three measurements.

Different types of vehicles have different effects on the formation and characteristics of traffic. In order to take this influence into account, the reduction coefficients to a relative passenger car are used, which are determined by comparing their dynamic dimensions.

Based on the experimental studies, it was found that 3888 cars passed through the intersection during the period of record (2 hours), the value was reached on Wednesday from 5:00 p.m. to 7:00 p.m. Peak traffic intensity was 1944 cars / hour.

There is congestion when turning left from Polesskaya Street to Generala Rodina Street and when turning left from Generala Rodina Street to Polesskaya Street as a result of traffic congestion.

In the course of simulation, a scaled map of the intersection of Generala Rodina Street Polesskaya Street was entered, as well as transport infrastructure elements and the results of experimental studies on traffic intensity.

As a result of the simulation, the function of the output $Z(t)$ parameter describing the state of the roadway was obtained. Based on the results of mathematical and simulation modeling, the necessity of introducing a separate lane for turning right from Generala Rodina Street to Polesskaya Street, as well as broadening Generala Rodina Street at the intersection, is noted.

The function of the output parameter also describes the optimal duration of the traffic signalization phases in the considered section of the RN, which can be implemented with adaptive control of traffic flows within the ITS.

\section{Conclusion}

The proposed mathematical and simulation models allow us to optimize the process of traffic management, which will positively affect traffic safety. The adequacy of the proposed models is confirmed by the results of experimental studies conducted at the intersection of Generala Rodina and Polesskaya Streets in the city of Orel. As a result of the proposed measures to improve the road traffic organization, the traffic capacity of the intersection under consideration will increase by $20 \%$, and by minimizing the number of conflict points at the intersection, road safety will increase.

\section{References}

1. Bioengineering systems: Theory and Design / Edited by V.M. Akhutina.- L .: Publishing House of Leningrad State University, 1981. - p.220

2. Ventzel, E.S. Operations research. Tasks, principles, methodology. M.: Higher school, 2001. - p. 208.

3. Baranov, Yu.N. Fundamentals of security in the system "man - machine environment” [Text] / Yu.N. Baranov, A.A. Katunin, R.V. Shkrabak, Yu.N. Braginets // Vestnik of NTSBZHD. - 2014. - No. 1 (19). - p.p 73-76. 
4. Baranov, Yu.N. Factors determining the dangerous action of the driver when driving a vehicle [Text] / Yu.N. Baranov, D.O. Kozhin, D.E. Alyokminsky, V.V. Evgrashin // Collection of scientific papers Sworld. 2014.Vol. 2. No. 4. p.p 3-7.

5. Baranov, Yu.N. Study of the "M-M" system in the formation of production hazards [Text] / Yu.N. Baranov, R.V. Shkrabak, Yu.N. Braginets, P.A. Pantyukhin // Vestnik of St. Petersburg State Agrarian University, No. 26, 2012 - p.p 438-440.

6. Baranov, Yu. N. Logical-graphical analysis of the occurrence of dangers of a collision of vehicles during visual reflection of the process of their braking [Text] / Yu.N. Baranov, A.N. Zagorodnykh // Vestnik of Orel State Agrarian University. 2011.Vol. 29. No. 2. p.p 70-73..

7. Tryastsin, A.P. Formation of integrated technological safety of automotive systems [Text] / A.P. Tryastsin // World of transport and technological machines. 2013. No. 3 (42). p.p 89-94.

8. Zatvornitsky, A.P. Search algorithm for the optimal way in a road network, under conditions of uncertainty / V. E. Mezhov, A.P. Zatvornitsky, O.N. Cherkasov // Transport business of Russia. 2006. -Vol. 7. - p. 32.

9. Belokurov, V. P. The choice of solutions at search iterations in numerical vector schemes for modeling transport systems / V. P. Belokurov // Multidisciplinary network electronic scientific journal of Kuban State Agrarian University. - 2012.-No.76.-p.p 574-583

10. Novikov, A.N. Improving the road network to increase its traffic capacity using means of transport telematics [Text] / A.N. Novikov, V.A. Golenkov, Yu.N. Baranov, A.A. Katunin, A.S. Bodrov // Vestnik of Tula State University. Technical science. 2014. No. 6. p.p 128-139.

11. Baranov Yu.N. Calculation of probability measures of road safety by the method of models of Markov processes [Text] / Yu.N. Baranov, E.N. Khristoforov, N.E. Sakovich, A.M. Nikitin // World of Transport and Technological Machines. 2014. No. 4 (47). p.p 115-124

12. Novikov, A.N. Modernization of the road network of the city of Orel (on the example of the Naugorskoe highway) [Text] / A.N. Novikov, Yu.N. Baranov, A.A. Katunin, D.D. Matnazarov // World of Transport and Technological Machines. 2014. No. 2 (45). p.p 86-96.

13. Baranov, Yu.N. Traffic optimization on the example of the street-road network of the city of Orel [Text] / Yu.N. Baranov, D.O. Kozhin, D.E. Alyokminsky, V.V. Evgrashin // Vestnik of NTSBZHD. 2014. No. 3 (21). p.p 8-14.

14. Baranov, Yu.N. Quantitative assessment of human reliability in human-machine systems [Text] / Yu.N. Baranov, A.I. Pantyukhin, A.L. Kuznetsov // Vestnik of Orel State Agrarian University. 2010.Vol. 27. No. 6. p.p 139-140.

15. Baranov, Yu.N. Methodological approach to forecasting the danger and injury risk of agricultural workers [Text] / Yu.N. Baranov, A.I. Pantyukhin, Vestnik of Krasnoyarsk State Agrarian University. 2009. No. 8. p. 145.

16. Novikov, A.N, Optimization of local events for organizing traffic in the city of Orel using simulation computer methods [Text] / A.N. Novikov, Matnazarov D.D., V.A. Golenkov, A.A. Katunin / Alternative energy sources in the transport and technological complex: problems and prospects of rational use. 2014. No. 1. p.p 382-387.

17. Przhibyl, P. Transportation as a science [Text] / Przhibyl, P., A.N. Novikov, A.A. Katunin // World of Transport and Technological Machines. 2014. No. 3 (46). p.p 96109. 
18. Przhibyl, P., Svitek, M. Transport Telematics: Czech translation by O. Buzekai, V. Buzkova. Edited by prof.V. V. Silyanova -M .: MADI (STU), 2003 -p.540.

19. Katunin, A.A. Remote control of parameters of automobiles technical systems as part of ITS [Text] / A.A. Katunin, M.V. Peshekhonov // Automobile and Electronics. Modern technologies.-2013.No.2 (5).- p.p 114-118. 